\title{
Coexistence of scale invariant and rhythmic behavior in self-organized criticality
}

\author{
S. Amin Moosavi, 1,2 , * Afshin Montakhab, ${ }^{2}$ and Alireza Valizadeh ${ }^{1}$ \\ ${ }^{1}$ Department of Physics, Institute for Advanced Studies in Basic Sciences, Zanjan 45137-66731, Iran \\ ${ }^{2}$ Department of Physics, College of Sciences, Shiraz University, Shiraz 71946-84795, Iran
}

(Dated: July 25, 2018)

\begin{abstract}
Scale-free behavior as well as oscillations are frequently observed in the activity of many natural systems. One important example is the cortical tissues of mammalian brain where both phenomena are simultaneously observed. Rhythmic oscillations as well as critical (scale-free) dynamics are thought to be important, but theoretically incompatible, features of a healthy brain. Motivated by the above, we study the possibility of coexistence of scale-free avalanches along with rhythmic behavior within the framework of self-organized criticality. In particular, we add an oscillatory perturbation to local threshold condition of the continuous Zhang model and characterize the subsequent activity of the system. We observe regular oscillations embedded in well-defined avalanches which exhibit scale-free size and duration in line with observed neuronal avalanches. The average amplitude of such oscillations are shown to decrease with increasing frequency consistent with real brain oscillations. Furthermore, it is shown that optimal amplification of oscillations occur at the critical point, further providing evidence for functional advantages of criticality.
\end{abstract}

\section{INTRODUCTION}

Generic scale-invariance is ubiquitously observed in natural systems [1-3] and has been a subject of study in physical [4 6], geological [7], biological [16] and social sciences [8]. On the other hand, rhythmic behaviors such as resonance and synchronization 9 are also observed and studied in many natural systems [10]. From a theoretical point of view these two phenomena seem to be incompatible, since oscillations imply a definitive time-scale while scale-free avalanches exhibit no particular time (or size) scales.

A perfect example of a system where both phenomena are observed is the collective neural dynamics of mammalian cortex. On one hand, rhythmic oscillations of cortical neurons are well documented and intensively studied in regard to their formation [11 14 as well as their functional and behavioral correlates [15]. On the other hand, neuronal avalanches [16-22] with scale-free statistics of their size and duration imply lack of time and size scales for brain dynamics. It has been shown that coexistence of these two phenomena is important for development of cortical layers 23]. Scale-free behavior of the brain, that is thought to be the result of underlying criticality, has recently attracted much attention in regard to optimum dynamic range in response to stimulus [24, 25], functional robustness [26], learning capability [27], information processing [28] and transmission [29].

The important issue here is how these two phenomena, that seem to be incompatible in a first pass, emerge simultaneously in the cortex. Moreover, what would be the consequences of such a coexistence. Despite the importance of this phenomena, a few theoretical studies have been devoted to this subject. Poil et al. have shown that this phenomena can emerge as a result of a balance between inhibition and excitation [30]. More recently Wang et al. have shown that this coexistence can emerge as a finite-size effect in self-organized critical states of small systems 31]. Emergent stochastic oscillations are also observed close to the critical point in non-conservative systems of interacting excitable nodes known as self-organized quasi-critical models [32, 33].

The typical theoretical framework for the study of rhythms of the brain is synchronization of coupled phase oscillators such as the Kuramoto model [9] while neuronal avalanches are mostly studied in the context of self-organized criticality (SOC) [31, 34 39] or models of excitable nodes [25, 30, 40 42]. In this work, we intend to study the effects of oscillations in the framework of SOC. In particular, we introduce and justify an oscillatory perturbation into a well-known Zhang model of SOC [43, 44] and subsequently characterize its response to such a perturbation. Interestingly, we find that oscillations dominantly occur while embedded within well defined avalanches which exhibit scale-free statistics for their size and duration. We further find that the well established response of the system is further enhanced and amplified at the critical point leading to large amplitude oscillatory behavior as a result of subthreshold oscillatory perturbations.

\section{THE MODEL}

In order to study the behavior of a self-organized critical (SOC) model under the influence of an external oscillatory perturbation we use a sandpile model known as the stochastic Zhang sandpile model [44]. The reasons for choosing this model for our study is that it exhibits continuous dynamical variables, a threshold dynamics that can mimic the neuronal dynamics and wellbehaved scaling behavior [45]. The model is considered on a two dimensional $L \times L$ square lattice (number of sites in the lattice is $N=L^{2}$ ) with nearest neighbor 
interactions. Every node of the lattice $i$ is assigned a dynamical variable $V_{i}$ (e.g. energy or potential). Dynamics of the model exhibits a slow perturbative drive during which small amounts of energy are added to the system, i.e. $V_{i}=V_{i}+\delta V$ where $\delta V$ is a randomly chosen real number from $[0,0.25]$. This process is continued until a site reaches a threshold value $\left(V_{t h}=1\right)$ at this point a fast dynamics takes place. Addition of energy via perturbative drive is not possible during an avalanche in SOC systems. This property is known as separation of slow and fast time scales [4 6]. The rule for the fast dynamics is that each site $i$ with $V_{i} \geq V_{t h}$ becomes unstable and distributes its energy between its neighbors with the following toppling rule

$$
\begin{array}{ll}
\text { if } & V_{i}>V_{t h} \\
\text { then } & V_{j} \rightarrow V_{j}+W_{j} V_{i}, \quad V_{i} \rightarrow 0
\end{array}
$$

in which the index $j$ is related to all neighbors of site $i$, and $W_{j}$ are annealed random numbers in the range $[0,1]$ with the constraint of $\sum_{j} W_{j}=1$ which leads to strict local conservation of energy. Conservative dynamics in addition to separation of time-scales are believed to be required for observation of self-organized criticality. However, it has been shown that breaking local conservation does not violate criticality of the system as long as the dynamics is on the average (or globally) conservative [36, 37]. It is generally believed that neuronal interactions are not conservative where different electrophysiological mechanisms play role in delivering electrical signal via synapses. Therefore, models of nonconservative interacting neurons have been developed as self-organized quasi-critical models where approximate criticality is considered as an explanation of scale-free behavior of neuronal avalanches $32,34,46$. However, in this work we use a conservative SOC model which can be considered as a limiting case of a more realistic model of neuronal dynamics.

The fast dynamics pursuing Eq. (11) is triggered by a single toppling. As a result of a toppling it is possible that the neighbors that receive energy become unstable and topple in the next time step and a cascade of toppling takes place which is called an avalanche. An avalanche ends when there are no unstable sites in the system. Here we must note that all the sites that become unstable in one time step will topple together, i.e. we use the parallel update rule 47]. Boundaries of the lattice are open and energy can be dissipated through the boundaries. Size $(S)$ of an avalanche is defined as the number of topplings and duration $(D)$ of an avalanche is defined as the number of time steps (parallel updates) of the avalanche.

We also use another version of sandpiles known as fixed energy sandpile model [48, 49]. In this version, periodic boundary conditions are imposed on the system and the external perturbation is turned off. Therefore, the average energy of the system $\left(E=1 / N \sum_{i=1}^{N} V_{i}\right)$ is fixed by the initial conditions. Fixed energy sandpile models ex- hibit a control parameter which is the average energy of the system $E$ and an order parameter which is the long term average of density of active nodes $\rho$ in the system. Activity is initiated by choosing a random site $i$ and allowing it to topple according to Eq.(1), regardless of its value $V_{i}$. This model exhibits a continuous (dynamical) phase transition, passing through a critical point $E=E_{c}$, from an absorbing state where any activity ends $(\rho=0)$ to a running state where one observes ceaseless dynamics $(\rho>0)$. Properties of the system at the critical point of fixed-energy sandpile is in accordance with its SOC counterpart [48]. Using fixed-energy sandpiles, we can study the behavior of the system in the sub-critical $\left(E<E_{c}\right)$ as well as super-critical $\left(E>E_{c}\right)$ phases.

We now introduce an oscillatory perturbation to the system. During the fast dynamics we simply introduce a sub-threshold oscillatory perturbation to the model that changes the dynamics by changing the condition of toppling as follows:

$$
\begin{array}{ll}
\text { if } & V_{i}+\delta \times f\left(\Omega t+\phi_{0}\right)>V_{t h} \\
\text { then } & V_{j} \rightarrow V_{j}+W_{j} V_{i}, \quad V_{i} \rightarrow 0
\end{array}
$$

in which $f$ is a normalized oscillatory function, $\delta$ is the (sub-threshold) amplitude of the oscillatory perturbation, $\Omega=2 \pi / T$ is the angular frequency of the oscillations ( $T$ is the duration of oscillatory perturbation) and $\phi_{0}$ is the initial phase that is chosen randomly from $[0,2 \pi]$ at the beginning of each avalanche. Here, we must note that the oscillatory perturbation does not add energy to the system it just manipulates the condition of toppling and the dynamics is, regardless of the oscillations, strictly conservative. In this paper we will show that it is possible to introduce a time-scale to the dynamics of a critical system while it still remains at the critical point.

An example of a physical situation for our model is a cortical tissue that receives sub-threshold oscillatory input from any other parts of the brain. A real neuron undergoes oscillations in its membrane potential when receiving a sub-threshold oscillatory input current [50]. Subsequently, the excitability of a neuron becomes an oscillatory function of time. Therefore, our model captures in a simple way a threshold and release mechanism, driven by an external oscillatory plus local inputs, and thus resembles what one expects from real neuronal dynamics.

\section{RESULTS}

To understand the scaling as well as oscillatory behavior of the system, we performed extensive computer code simulations of the systems with different sizes of $L=512,1024,2048$. First, we focus on the activity of the system $(x)$ which is defined as the number of active sites (the sites that topple) at each time step of an avalanche. An avalanche starts when $x$ becomes equal to 

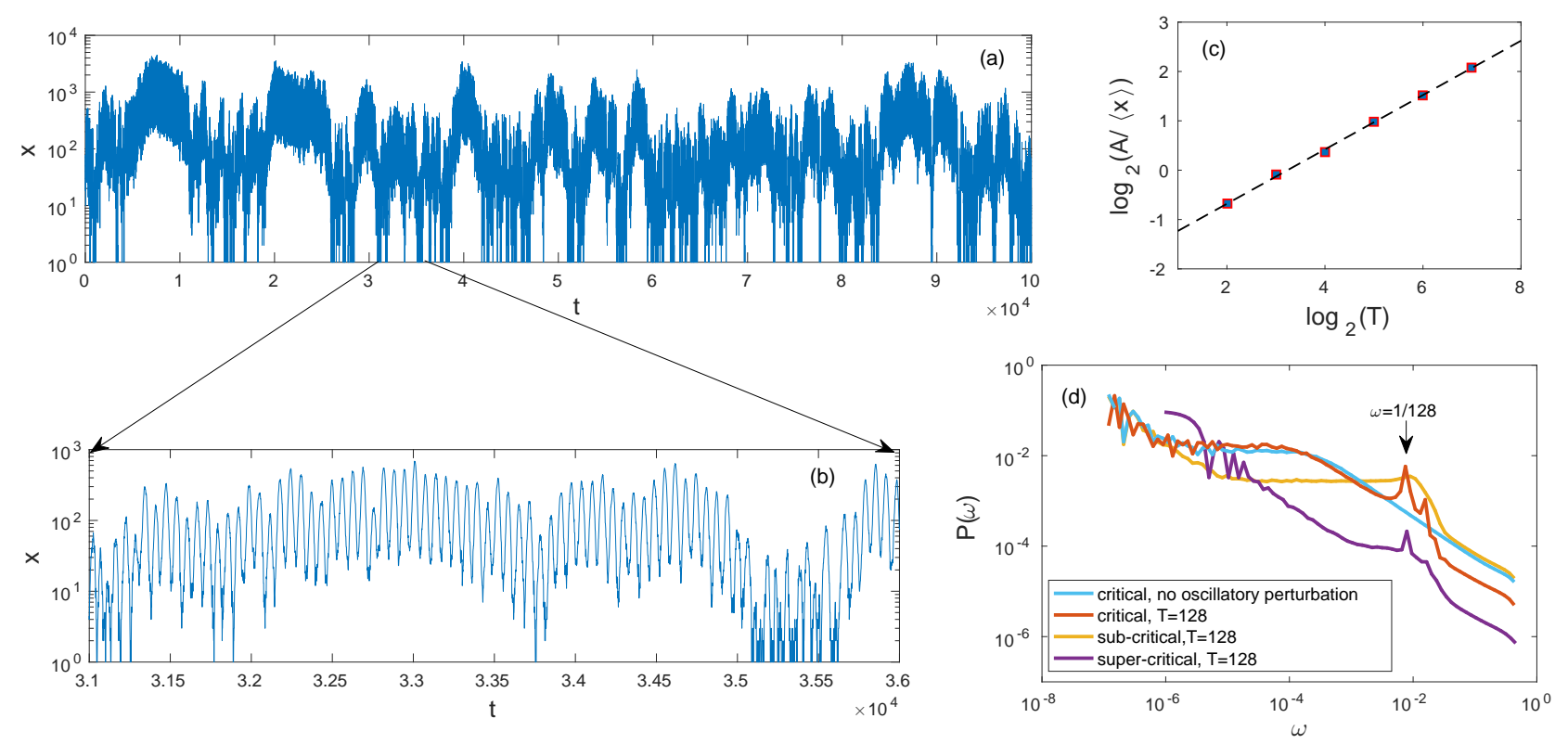

FIG. 1. (Color on line)(a) Activity of the system (consecutive avalanches) as a function of time for a system with $T=64$. An avalanche starts when $x>0$ and ends when $x=0$. Note the logarithmic scale on the vertical axis. (b) Oscillations of the activity embedded in avalanches. (c) Normalized amplitude of oscillations in activity as a function of period of oscillations. (d) Power spectrum of $x$ for different phases of systems with oscillatory perturbations with $T=128$ at $\left(E=E_{c}=0.596\right)$, above $(E=0.608)$ and bellow $(E=0.585)$ the critical point, compared with the critical system without oscillatory perturbation. We set $L=1024, f=\sin \left(\Omega t+\phi_{0}\right)$ and $\delta=0.1$ in all panels.

one and ends when it becomes zero. We find that systems that are influenced by the oscillatory perturbation exhibits oscillations in activity embedded in avalanches, i.e. during each avalanche $x$ is an oscillatory function of time with a period equal to the oscillatory perturbation (see Fig!(a,b)).

In order to study the properties of oscillatory perturbations we calculate the average amplitude of oscillations as

$$
A=\left\langle A_{k}\right\rangle=\left\langle\left\langle x_{\text {max }}^{k}\right\rangle-\left\langle x_{\text {min }}^{k}\right\rangle\right\rangle
$$

where $\left\langle x_{\max }^{k}\right\rangle$ and $\left\langle x_{\min }^{k}\right\rangle$ are respectively the average of maxima and minima of oscillatory activity in an oscillatory avalanche $k$. Large brackets are for averaging over all oscillatory avalanches. An interesting property of these oscillations is that their normalized amplitude increases as a power-law function $\left(A /\langle x\rangle \sim T^{0.55}\right.$ where $\langle x\rangle$ is the average activity of the system over all oscillatory avalanches) of the period of oscillatory perturbation (see Fig1(c)). This property is in agreement with our knowledge of the rhythms of the brain [11] where we observe low amplitude, high frequency oscillations and vice versa. For example, low frequency alpha $(\sim 10 \mathrm{~Hz})$ oscillations occur at relatively high amplitude while high frequency gamma $(30-80 \mathrm{~Hz})$ oscillations occur at low amplitudes in the cortex.
Power spectrum of the activity of system is calculated and plotted in Fig 1(d) for different fixed-energy models with and without oscillatory perturbations at $E=E_{c}$, and also above and bellow the critical point. In the subcritical phase we observe a flat line for low frequencies which is an indication of a noisy dynamics. Comparing to the critical state of the system without oscillatory perturbation, it is clear that for the critical system with oscillatory perturbation, one obtains a power-law function with a peak at the frequency of oscillatory perturbation. This behavior is ubiquitously observed in electroencephalogram as well as local field potential analysis of many parts of the brain [51]. We have done the same analysis for different values of $T=128,64,32$ and we observed the same behavior in all cases. Therefore, we can conclude that the system exhibits a wide range of frequencies that have the potential to be amplified and observed at the critical point. A degree of amplification of oscillatory perturbations is observed in the super critical phase with an amplitude smaller than the the observed amplitude in the critical phase (see Fig.1(d)). Using a quantitative analysis, we will show later that the amplification is maximized at the critical point compared to off-critical phases. We note that in contrast to the phenomenon of resonance where amplification of special frequencies is possible, this amplification is possible for all the power-law distributed frequency range. This behavior is in line with oscillatory behavior in the brain 
where a wide range of frequencies is observed [11, 51].

In order to study the scaling behavior of the system we focus on avalanche statistics. A prototypical example of probability distribution function of duration of avalanches $(P(D))$ is plotted in Fig 2(a) for $L=512$, $f=\sin \left(\Omega t+\phi_{0}\right), \delta=0.1$ and $T=128$. It is interesting that $P(D)$ exhibits a bouncing behavior for large enough $D$ over intervals of $\Delta D=T$. And, if we average the data over time bins of $\Delta D=T$, we observe power-law behavior of the binned data. Notably, this bouncing behavior is not observed for the probability distribution function of avalanche sizes and a standard power-law behavior is observed for $P(S)$.

Simply observing extended scaling for a finite system is not necessarily proof of criticality. To verify establishment of self-organized criticality and also evaluating scaling exponents, we perform a finite-size scaling of our data for different system sizes of $L=512,1024,2048$. We consider a simple scaling ansatz for the probability distribution function of size and duration of avalanches:

$$
P(y) \sim y^{-\tau_{y}} G_{y}\left(y / L^{\beta_{y}}\right)
$$

where $y$ can be either the binned data of $D$ or $S, \tau_{y}$ is the critical exponent, $\beta_{y}$ is the finite size exponent determining the cutoff in $P(y)$, and $G_{y}$ is the universal function that, in the case of criticality, exhibits the same shape for all system sizes [4]. If the system is critical, and we rescale $y \rightarrow y / L^{\beta_{y}}$ and $P(y) \rightarrow y^{\tau_{y}} P(y)$, then the plots of rescaled data must collapse into one universal curve for different system sizes. In Fig 2 (c,d) we present the results of finite-size scaling analysis of our data. We observe good collapse of data for both cases of $D$ and $S$ with $\tau_{S}=1.28(1), \tau_{D}=1.50(1)$, $\beta_{S}=2.75(1)$ and $\beta_{D}=1.50(1)$. These values of exponents are in agreement with the scaling exponents in the absence of oscillatory perturbations [4, 36, 49], which indicates robustness of scaling behavior of SOC systems in the presence of oscillatory perturbations. Here, we can conclude that despite having oscillatory behavior the system exhibits scale invariance and criticality. We note that the same analysis were performed for different $f=\sin \left(\Omega t+\phi_{0}\right), f=\sin \left(\Omega t+\phi_{0}\right)+1, f=\sin \left(\Omega t+\phi_{0}\right)-1$ as well as different values of $\delta=0.5,0.1,0.2$ and the same behavior is observed in all cases.

To better understand the behavior of the system in sub- and super-critical phases we performed computer simulations of the above explained fixed-energy sandpile model with $L=1024, T=64,128$ and $\delta=0.0,0.1$. Fig [3(a) is a plot of the order parameter $\rho$ versus the control parameter $E$. It is clearly seen that there is a continuous phase transition at the critical point $E_{c}$ which is in agreement with the $E_{c}$ that is obtained from the selforganization process in the standard form of the model with open boundary conditions and external drive (see Fig $3(b))$.
The key feature of neural networks poised close to a standard second order phase transitions is "optimum" response to stimulus [25, 28]. So far, we have essentially added small subthreshold oscillations to a well-known model of SOC, and have characterized their effect on the activity of the system. We next ask to what extend criticality provides amplifications of such oscillations. In order to quantify the amplification of oscillatory perturbations, we define $\chi$ as the expectation value of average amplitude of oscillations over all active times

$$
\chi=\left\langle P_{k} \frac{A_{k}}{\langle x\rangle_{k}}\right\rangle
$$

where $P_{k}$ is the probability of having a rhythmic behavior in an avalanche $k$, which is a binary probability, i.e. it is equal to one if there is an oscillation in the avalanche and is equal to zero otherwise. $A_{k}$ is the average amplitude of oscillations as defined in Eq.(3) and $\langle x\rangle_{k}=S_{k} / D_{k}$, is the average activity during the $k^{t h}$ avalanche, which serves as normalization. Large brackets indicate averaging over all avalanches. Fig.3(c) shows a plot of $\chi$ around the critical points as a function of $E-E_{c}$ for $T=64,128$. It is interesting that amplification of oscillations is maximized at the critical point regardless of the value of $T$. However, we observe larger amplification for slower oscillations around the critical point which is in agreement with our results of Fig 1(c). We therefore conclude that optimal amplification of sub-threshold oscillations occurs at criticality. This has important consequences for brain function as production of rhythms are thought to be key elements in coding and transfer of information in the brain. This yet provides another motivation for the critical brain hypothesis.

\section{CONCLUDING REMARKS}

Motivated by critical as well as oscillatory dynamics of neuro-cortical circuits, we have analyzed a simple model of SOC which is influenced by sub-threshold oscillations. Interestingly, we find that the system exhibits well-defined oscillations embedded in avalanches where the average amplitude of oscillations is an increasing power-law function of the period of oscillations. Consequently, an off-critical system, that exhibits a time scale $D_{\max }$ for avalanches, cannot respond to a wide range of frequencies and is thus limited in range of oscillatory activity it can exhibit. However, due to scale-free behavior of avalanches at the critical point one observes a proper response to all frequencies. This could be important for a functional brain since we observe a wide range of frequency of rhythms in different regions of the brain.

One might be tempted to associate the observed amplification of system's response to stochastic resonance (SR), as there too, one observes the amplification of external (subthreshold) drive frequency in a stochastic 

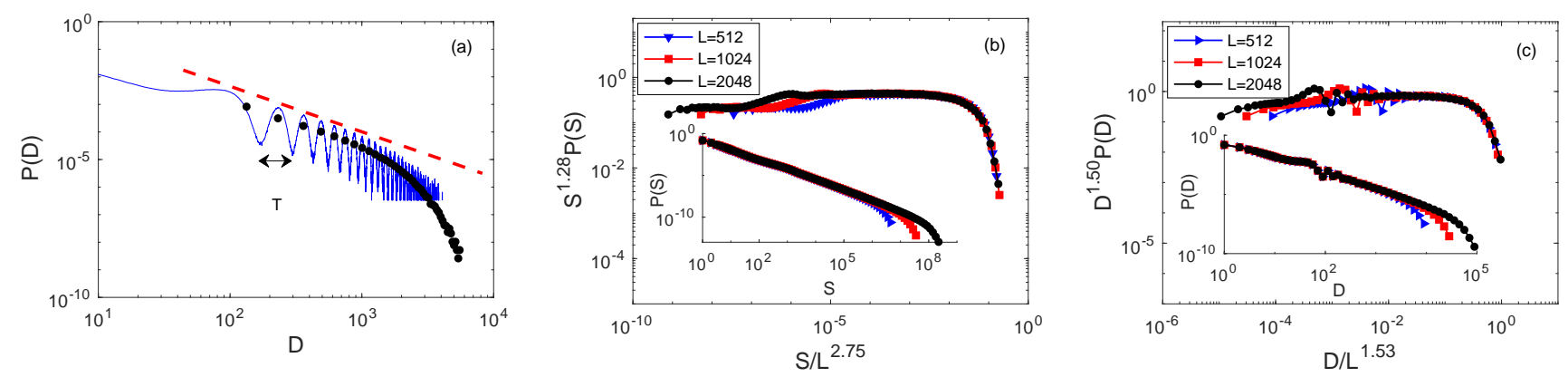

FIG. 2. (Color on line) (a) Probability distribution function of duration of avalanches $(P(D))$ is plotted for $L=512, f=$ $\sin \left(\Omega t+\phi_{0}\right), \delta=0.1$ and $T=128$. Circles are the results of averaging over intervals of $\Delta D=T$ which exhibit power-law behavior shown by the dashed line. (b,c) Main panels are finite-size scaling collapse of rescaled data into one universal curve for probability distribution functions of duration $(D)$ and size $(S)$ of avalanches where $\tau_{S}=1.28$ and $\tau_{D}=1.50$. Insets are plots of probability distribution functions of $S$ and $D$ corresponding to the main panels.
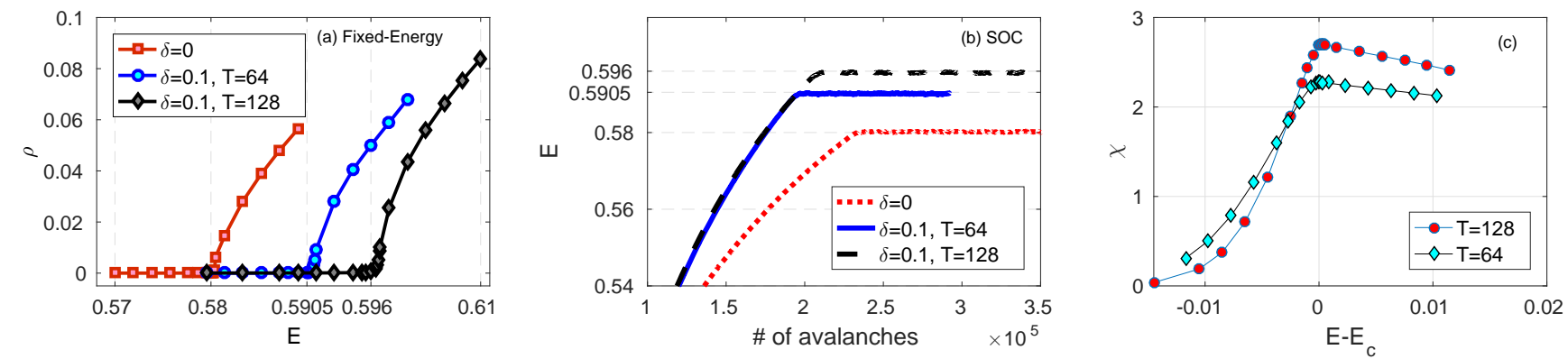

FIG. 3. (Color on line) (a) Second order (continuous) phase transition from absorbing to running state for fixed energy sandpile models with oscillatory perturbations. (b) Self-organization of average energy where after a transient time the open system with external drive settles into the critical steady state in agreement with the transition point of the fixed-energy model shown in panel (a). (c) Average amplitude of oscillations as a function of the control parameter $E$.

background. However, the mechanisms are entirely different. The criticality associated with the collective dynamics of our model is capable of amplifying a wide range of subthreshold frequencies, without need to tune any system parameter. On the other hand, in SR one needs to tune the noise intensity (and thus system's natural frequency) in order to see amplification in response for a given frequency.

Our finite size scaling analysis of the statistics of size and duration of avalanches suggests that, despite having oscillations, the system exhibits critical properties in agreement with the systems without oscillatory perturbations. We showed that the same exponents are observed with oscillatory perturbation and thus the robustness of criticality as well as universality class was confirmed. We note that divergence of avalanche durations with system size implies that duration of spontaneous oscillations in the resting state of the brain should only be bounded by the size of the cortex.

We also note that the exponents we obtained for the Zhang model are not the same as the standard meanfield exponents for real neuronal avalanches. However, on can imagine structural as well as dynamical modifica- tions to our model which could lead to mean-field exponents. For example, our $2 D$ nearest neighbor interaction is not a good topology for the real cortex. Larger average connectivity along with random neighbor would lead to mean field behavior, which is the exact solution for an allto-all network model. Furthermore, as has been shown in Ref. [36], addition of synaptic noise in the dynamics could also lead to mean field behavior independent of the structure of the network chosen.

It has been shown that criticality of the brain leads to many advantages for the brain functions 24 29]. Due to the crucial role of oscillations in brain functions, it is very important that the brain respond to oscillatory perturbations efficiently. We show that the optimum amplification of oscillatory perturbations takes place at the critical point. In other words, not only the system remains critical but also amplification of oscillations is allowed over a wide range of frequencies. This optimum amplification can be the root to optimum signal coding and transmission by oscillations over different time and length scales.

Support of Iranian National Elites Foundation, Institute for Advanced Studies in Basic Sciences, and Shiraz University Research Council is acknowledged. S.A.M. 
also acknowledges Y. Sobouti without whose support this work could not be completed.

* moosavi.s.amin@gmail.com

[1] B. B. Mandelbrot, The Fractal Geometry of Nature, (Freeman, New York 1983).

[2] L. P. Kadanoff, Phys. Today 39, 6 (1986).

[3] P. Bak, How Nature Works: the Science of Self-Organized Criticality (Springer-Verlag New York, 1996).

[4] G. Pruessner, Self-Organized Criticality: Theory, Models and Characterization (Cambridge University Press New York, 2012).

[5] P. Bak, C. Tang, and K. Wiesenfeld, Phys. Rev. Lett. 59, 381 (1987).

[6] P. Bak, C. Tang, and K. Wiesenfeld, Phys. Rev. A 38, 364 (1988).

[7] B. Gutenberg, and C. F. Richter, Seismicity of the Earth and Associated Phenomena, (Princeton University Press, Princeton 1954).

[8] C. J. Rhodes, and R. M. Anderson, Nature 381, 600 (1996).

[9] J. A. Acebrn, L. L. Bonilla, C. J. Prez Vicente, F. Ritort, and R. Spigler, Rev. Mod. Phys. 77, 137 (2005).

[10] A. Pikovsky, M. Rosenblum, and J. Kurths, Synchronization: a universal concept in nonlinear sciences, (Cambridge university press,2003).

[11] G. Buzsaki, Rhythms of the Brain (Oxford University Press, 2006).

[12] X. J. Wang, and G. Buzsaki, J. neurosci. 16, 6402 (1996).

[13] A. Compte, M.V. Sanchez-Vives, D.A. McCormick, and X-J Wang, Journal of neurophysiology 89, 2707 (2003).

[14] S. Sadeghi, and A. Valizadeh. Journal of computational neuroscience 36, (2014).

[15] X. J. Wang, Physiological reviews. 90, 1195 (2010).

[16] J. M. Beggs, and D. Plenz, J. Neurosci. 23, 11167 (2003).

[17] J. M. Beggs, and D. Plenz, J. Neurosci. 24, 5216 (2004).

[18] E. Tagliazucchi, P. Balenzuela, D. Fraiman, and D. R. Chialvo, Front. Physiol. 3, 15 (2012).

[19] N. Friedman, S. Ito, B. A. W. Brinkman, M. Shimono, R. E. Lee DeVille, K. A. Dahmen, J. M. Beggs, and T. C. Butler, Phys. Rev. Lett. 108, 208102 (2012).

[20] T. Petermann, T. C. Thiagarajan, M. A. Lebedev, M. A. L. Nicolelis, D. R. Chialvo, and D. Plenz, PNAS, 106, 37 (2009).

[21] O. Shriki, J. Alstott, F. Carver, T. Holroyd, R. N. A. Henson, M.L. Smith, R. Coppola, E. Bullmore, and D. Plenz, J. Neurosci. 33, 7079 (2013).

[22] D. Chialvo, Nature Physics 6, 744 (2010).

[23] D. Gireesh Elakkat, and D. Plenz. PNAS 105, 7576 (2008).

[24] W. L. Shew, H. Yang, T. Petermann, R. Roy, and D. Plenz. J. Neurosci. 29, 15595 (2009).

[25] O. Kinouchi, and M. Copelli. Nature Physics 2.5, 384 (2006).

[26] A. Goodarzinik, M. D. Niry, and A. Valizadeh, BMC Neuroscience, 17, p41 (2016).
[27] L. de Arcangelis, and H. J. Herrmann. PNAS 107, 3977 (2010).

[28] J. M. Beggs, Philos. Trans. R. Soc. A 366, 329 (2008).

[29] W. L. Shew, H. Yang, S. Yu, R. Roy, and D. Plenz. J. Neurosci. 31, 55 (2011).

[30] S. S. Poil, R. Hardstone, H. D. Mansvelder, and K. Linkenkaer-Hansen, J. Neurosci. 32.29, 9817 (2012).

[31] Sheng-Jun Wang, G. Ouyang, J. Guang, M. Zhang, K.Y. M. Wong, and C. Zhou, Phys. Rev. Lett. 116, 018101 (2016).

[32] A. A. Costa, L. Brochini, and O. Kinouchi, Entropy, 19, 399 (2017).

[33] O. Kinouchi, L. Brochini, A. A. Costa, J. G. F. Campos, M. Copelli, arXiv:1803.05537.

[34] A. Levina, J. M. Herrmann, and T. Geisel. Nature Physics 3.12, 857 (2007).

[35] J. A. Bonachela and M. A. Muñoz, J. Stat. Mech. (2009) P09009.

[36] S. A. Moosavi, A. Montakhab, Phys. Rev. E 89, 052139 (2014).

[37] S. A. Moosavi, A. Montakhab, Phys. Rev. E 92, 052804 (2015).

[38] L. Brochini, A. de Andrade Costa, M. Abadi, A.C. Roque, J. Stolfi and, O. Kinouchi, Scientific reports 6, 35831 (2016).

[39] J. Hesse, T. Gross, Front, Syst. Neurosci. 8, 116, (2014).

[40] D. B. Larremore, W. L. Shew, E. Ott, F. Sorrentino, and J. G. Restrepo, Phys. Rev. Lett. 112, 138103 (2014).

[41] A. Levina, J. M. Herrmann, and T. Geisel, Phys. Rev. Lett. 102, 118110 (2009).

[42] S. A. Moosavi, A. Montakhab, and A. Valizadeh, Scientific reports 7, 7107 (2017).

[43] Y. C. Zhang, Phys. Rev. Lett. 63, 470 (1989).

[44] R. Pastor-Satorras, and A. Vespignani, Eur. Phys. J. B 18, 197 (2000).

[45] Some standard SOC models including the much studied BTW model exhibit multi-scaling with unusual critical behavior, see for example, A. Abdolvand and A. Montakhab, Eur. Phys. J. B 76, 21-30 (2010).

[46] J. A. Bonachela, S. De Franciscis, J. J. Torres, and M. A. Munoz. J. Stat. Mech. P02015 (2010).

[47] A sequential update rule is also possible. Differences between parallel and sequential update rules and their effects on the critical properties of sandpile models are discussed in references [4, 44].

[48] A. Montakhab, J. M. Carlson. Phys. Rev. E 58, 5608 (1998).

[49] A. Vespignani, R. Dickman, M. A. Munoz, and S. Zapperi, Phy. Rev. E 62, 4564 (2000).

[50] W. Gerstner, W. M. Kistler, R. Naud, and L. Paninski. Neuronal dynamics: From single neurons to networks and models of cognition, (Cambridge University Press, 2014).

[51] B. J. He, J. M. Zempel, A. Z. Snyder, and M. E. Raichle, Neuron 66, 353 (2010).

[52] Z. Gingl, L. B. Kiss, and F. Moss, Europhys. Lett. 29, 191 (1995).

[53] L. Gammaitoni, P. Hänggi, P. Jung, and F. Marchesoni Rev. Mod. Phys. 70, 223 (1998). 